\title{
Prevalence and associated factors of malaria among pregnant women in Sherkole district, Benishangul Gumuz regional state, West Ethiopia
}

\author{
Girma Bekele Gontie ${ }^{1}$, Haileab Fekadu Wolde ${ }^{2^{*}}$ and Adhanom Gebreegziabher Baraki ${ }^{2}$
}

\begin{abstract}
Background: Malaria during pregnancy leads to serious adverse effects on mothers and the fetus. Approximately 25 million pregnant women in sub-Saharan Africa live at risk of malaria. This study would help to achieve Sustainable Development Goals (SDGs) by improving programs that deal with the prevention of malaria. Therefore, this study aimed to assess the prevalence and associated factors of malaria among pregnant women.

Methods: A community-based cross-sectional study was conducted from July to August 2018 in Sherkole district, West Ethiopia. A multi-stage sampling technique was used to select 504 pregnant women. The intervieweradministered semi-structured questionnaire was used for data collection. Malaria was also diagnosed using a rapid diagnostic test. The data was entered using EPI info version 7.2.2.2 and transferred to SPSS version 20 for analysis. Descriptive statistics were done using frequency and percentages. Both bivariable and multivariable logistic regression models were employed. Variables having $p$-value $<0.2$ were included in the final multivariable model. Variables having $p$-values $<0.05$ from the multivariable model were considered to be significantly associated with the dependent variable. The adjusted odds ratio with its $95 \%$ confidence interval $(\mathrm{Cl})$ was used as a measure of association.

Results: Of the total 498 pregnant women who participated in this study, $51(10.2,95 \%$ Cl: $7.72-13.24)$ were found to have malaria. Of these, 46 (90.2\%) and 5 (9.8\%) were caused by Plasmodium falciparum and Plasmodium vivax, respectively. Decreasing Age (Adjusted Odds Ratio (AOR) 0.78; 95\% Cl 0.67-0.911), not using insecticide-treated bed net (ITN) (AOR 12.5; 95\% Cl 4.86-32.21), lack of consultation and health education about malaria prevention (AOR 7.18; 95\% Cl 2.74-18.81), being on second-trimester pregnancy (AOR 7.58; 95\% Cl 2.84-20.2), gravidae II (AOR 5.99; 95\% Cl 1.6821.44) were found to be significantly associated with malaria during pregnancy.

Conclusion: Malaria is still a public health problem among pregnant women in the Sherkole district. Age, ITN use, gravidity, gestational age, and health education had a significant association with malaria. Screening pregnant women for asymptomatic malaria infection and educating and consulting on the appropriate malaria preventive methods shall be provided.
\end{abstract}

Keywords: Malaria, Pregnant women, Benishangul Gumuz, Ethiopia

\footnotetext{
* Correspondence: haileabfekadu@gmail.com

${ }^{2}$ Department of Epidemiology and Biostatistics, Institute of Public Health, College of Medicine and Health Sciences, University of Gondar, Gondar, Ethiopia

Full list of author information is available at the end of the article
}

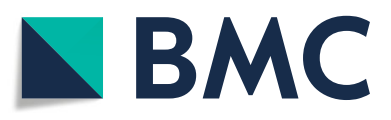

( ) The Author(s). 2020 Open Access This article is licensed under a Creative Commons Attribution 4.0 International License, which permits use, sharing, adaptation, distribution and reproduction in any medium or format, as long as you give appropriate credit to the original author(s) and the source, provide a link to the Creative Commons licence, and indicate if changes were made. The images or other third party material in this article are included in the article's Creative Commons licence, unless indicated otherwise in a credit line to the material. If material is not included in the article's Creative Commons licence and your intended use is not permitted by statutory regulation or exceeds the permitted use, you will need to obtain permission directly from the copyright holder. To view a copy of this licence, visit http://creativecommons.org/licenses/by/4.0/. The Creative Commons Public Domain Dedication waiver (http://creativecommons.org/publicdomain/zero/1.0/) applies to the data made available in this article, unless otherwise stated in a credit line to the data. 


\section{Background}

Malaria is caused by parasites of the genus Plasmodium and transmitted by female Anopheles mosquitoes. There are five different human malaria species such as $P$. falciparum, P. vivax, P. malariae, P. knowlesi and P. ovale. In 2016, an estimated 216 million cases of malaria and 445,000 deaths occurred worldwide [1]. Most, (90\%), malaria cases and 91\% of all malaria death in 2015 and 2016 were reported from the WHO African Region. Of the 91 countries reporting indigenous malaria cases worldwide, around $80 \%$ of the total cases were from subSaharan African countries [1, 2].

Malaria during pregnancy is a serious public health problem in sub-Saharan Africa. It is estimated that each year approximately 25 million pregnant women in sub-Saharan Africa live at risk of $P$. falciparum malaria infection [3]. Two institution-based studies done among pregnant women attending antenatal care (ANC) in Nigeria showed the prevalence of malaria to be $41.6 \%$ [4] and 7.7\% [5]. Another institution based study in Eastern Sudan showed $13.7 \%$ of pregnant women were infected with P. falciparum [6]. Studies conducted in Burkina Faso [7], and Malawi [8] also showed the prevalence to be $18.1 \%$, and $19 . \%$ respectively. Besides, two institution and one community-based studies conducted in different parts of Ethiopia also showed the prevalence of malaria among pregnant women to be between 2.83 and $16.3 \%$ [9-11].

Malaria infection during pregnancy causes an enormous risk to the mother, fetus, and neonates [12]. Indeed although malaria during pregnancy might be asymptomatic due to a high level of acquired immunity in mothers residing in high transmission areas, it is still associated with an increased risk of maternal anemia, spontaneous abortion, stillbirth, prematurity, and low birth weight $[3,13,14]$. Moreover, severe maternal anemia increases the mother's risk of death. Malariarelated anemia is estimated to cause as many as 10,000 maternal deaths each year in Africa [15].

Different risk factors for malaria among pregnant women were identified by previous studies. These include educational status $[7,16]$, age $[5,17]$, ANC visit, gestational age [18], parity [7, 18], gravidity, and ITN utilization [11].

In Benishangul Gumuz regional state, almost all districts $(98 \%)$ of the landmass are malarious areas and $97 \%$ of the population are at risk for malaria infection. Despite the high risk of malaria transmission in the area, there is limited evidence about the burden and risk factors of malaria among pregnant women which can be used for reducing maternal and child mortality due to the disease. Therefore, this study aimed to assess the prevalence of malaria and its associated factors among pregnant women in Sherkole District, Benishangul Gumuz regional state, West Ethiopia.

\section{Methods}

\section{Study area and period}

The study was conducted in Sherkole district, Benishangul Gumuz Regional state (BGRS) from July 20 to August 30, 2018. Sherkole district is one of the 21 BGRS administration districts which is found $756 \mathrm{~km}$ to the West of Addis Ababa, the capital city of the country, and $96 \mathrm{~km}$ far away from region city, Assosa. The district is found at a latitude of 13.169308 and longitude of 39.987117 and the altitude of the district is $680-800 \mathrm{~m}$ above sea level. The climatic condition of the district is hot and the annual temperature is estimated to be between $25^{\circ} \mathrm{C}$ and $41{ }^{\circ} \mathrm{C}$. The Annual range of rainfall in the district is $900-1200 \mathrm{~mm}$. In this district, all kebeles are malarious with 39,373 populations at risk of the disease. In 2016/2017, the annual malaria incidence rate in the district was 263 cases per 1000 population. There were 1243 pregnant women, 1196 under 1 year, and 6370 under 5 years old of children in the district (Fig. 1).

\section{Study design and population}

A community-based cross-sectional study was conducted. The source population for this study was all pregnant women at any gestational age living in the district. The study population was those pregnant women in the selected kebeles and who were available during the data collection period. Pregnant women with mental illness and severely debilitating diseases were excluded from the study.

\section{Sample size determination and sampling procedure}

The sample size was determined using a single proportion formula using a $50 \%$ prevalence of malaria among pregnant women, 95\% confidence level, 5\% margin of error, and design effect of 2 . To compensate for the non-response rate, $10 \%$ of the determined sample size was added. Finally, finite population correction was done to adjust the final sample size which gives a total sample size of 504. A multi-stage sampling technique was used to select the determined sample size. At the first stage, from a total of 20 kebeles in the district, 8 kebeles with a a total of 1243 pregnant women were selected by using a simple random sampling technique. In the second stage, the sample size was distributed proportionally for the 8 kebeles based on the number of pregnant women in the kebeles with a range of 41 to 79 housholds for each kebele and then households were selected using a simple random sampling technique. Finally, pregnant women in the household were taken and in the presence of more than one eligible woman in a single household, a lottery method was used to select one.

\section{Variable measurement and data collection procedure}

The outcome variable for this study was malaria infection which was assessed using RDT and pregnant 


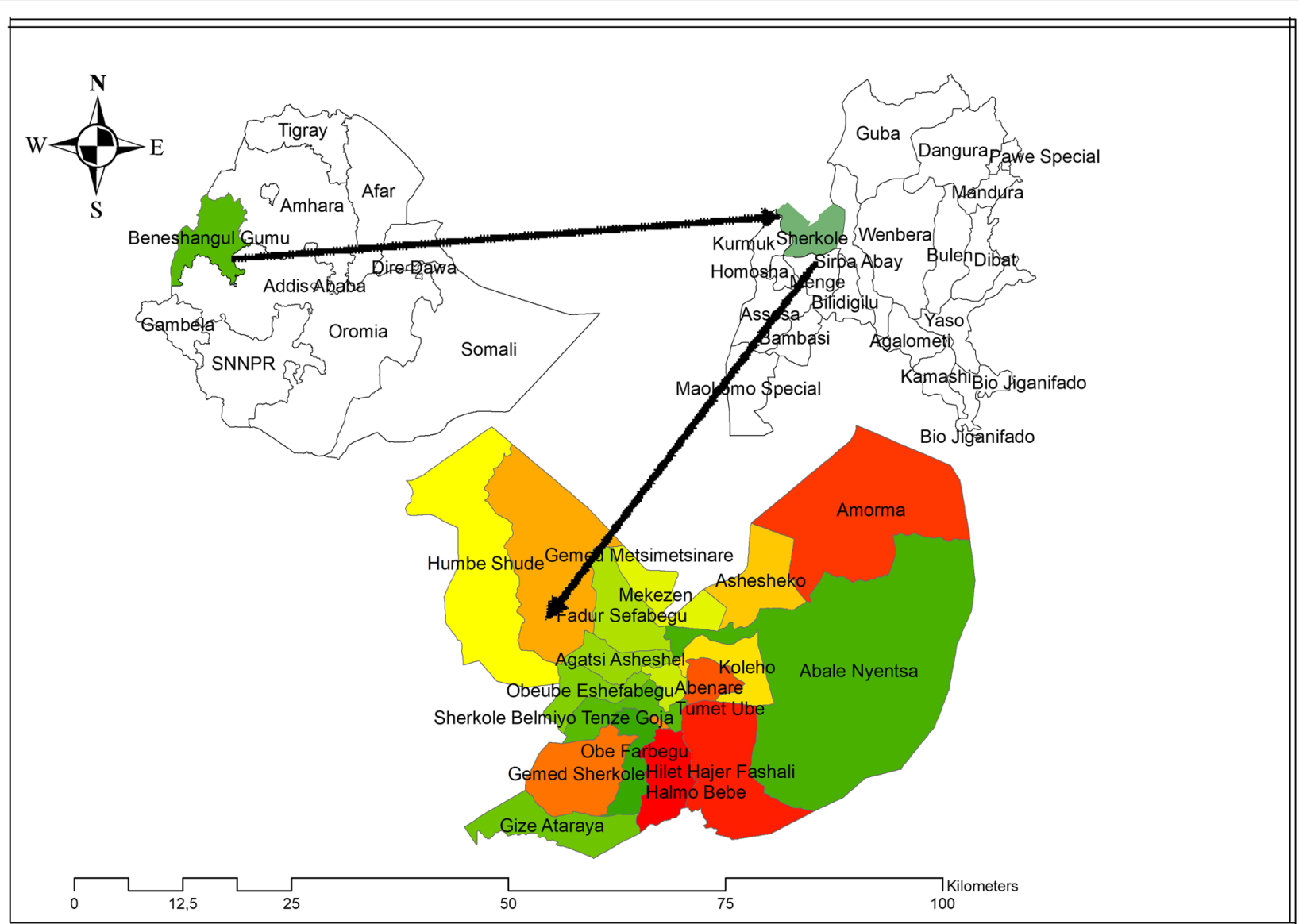

Fig. 1 Location of the study area

mother with any type of Plasmodium species from the test were considered as having malaria infection. The independent variables include socio-demographic factors (age, marital status, educational status, and occupational status); obstetric factors (gravidity, parity, trimester of pregnancy, history of abortion); malaria prevention measures (ITN ownership, indoor residual spraying (IRS) use, personal protective measures, and ITN utilization); health service use (accessibility of ANC, gestational age at the first visit, number of ANC visit, place of delivery for the previous child, previous history of malaria infection during pregnancy, and health education about malaria prevention methods during ANC follow up).

The interviewer-administered Semi-structured questionnaire was used to collect the required information. For those pregnant women who were on ANC followup, the data collector reviewed their antenatal followup cards to cross-check the information given by them. Card information checked includes; gravidity, parity, and gestational age at first ANC visit. Following the interviews, blood was obtained from the third finger of women's left hand. First, the tip of the finger was wiped with a piece of cotton wool lightly soaked in alcohol.
Then piercing with sterile lancet was done and the blood allowed to flow freely without squeezing the finger. Then, $5 \mu \mathrm{l}(\mu \mathrm{l})$ blood was collected and a single small drop was added on the CareStart RDT to examine the presence or absence of malaria and to differentiate its species. The RDT read and determine the species qualitatively after 15-20 min of putting the blood to the kit. Ten percent of the randomly selected negative slides were rechecked and reread. Eight trained diploma nurses and midwives collected the data and they were supervised by two health professionals with a qualification of BSc degree. The questioner was pretested and one-day training was given for supervisors and data collectors on the basic technique of the data collection.

\section{Data processing and analysis}

The data were entered using EPI-Info 7.2.2 and then transferred to SPSS version 20 statistical package for further analysis. Data cleaning and management were done. Descriptive statistics (frequencies, mean, SD, and percentage) were done to explain the study population in relation to relevant variables. The Chi-square assumption was checked for all categorical independent 
variables and multicollinearity was also checked using the Variance inflation factor (VIF). Both bi-variable and multi-variable logistic regressions were used to assess the association between outcome and explanatory variables. Factors with $p$-value $\leq 0.2$ from the bi-variable model were included in the final model. Variables having a $p$-value $<0.05$ from the multivariable model were considered as having a statistically significant association with the outcome. Adjusted Odds ratio with 95\% CI was used as a measure of association. The model goodness of fit was assessed using the Hosmer lemisho test.

\section{Result}

\section{Socio-demographic characteristics, obstetric} characteristics, and malaria prevention methods adopted by pregnant women

A total of 498 pregnant women participated in this study with a response rate of $98.8 \%$. The majority, 208(41.8\%), of the pregnant women were in the age group of 25-29 years. Concerning the educational status, more than three fourth, 384(77.1\%), of the mothers had no formal education. Almost all, 482 (96.8\%), study participants were farmers and traditional gold miners. About 478 (96\%) of respondents owned at least one mosquito bed net, and $405(81.3 \%)$ of them sleep under mosquito nets in the previous night. Almost all, 485 (97.8\%), of the households had Indoor Residual Spray (IRS) in the last 12 months. All women 431 (86.5\%) who had ANC follow-up were given health education about the prevention methods of malaria infection during their ANC follow-up. The majority, 323 (64.9\%), of the study participants were multi-gravida, and more than half, 292 (58.6\%), of the study participants were in their third trimester of pregnancy (Table 1).

\section{Prevalence of malaria infection among pregnant women} In our study, the prevalence of malaria was found to be 10.2\% (95\% CI: 7.72-13.24). Of these, 46(90.2\%) were $P$. falciparum cases and 5 (9.8\%) were $P$. vivax cases. From the total confirmed cases, the majority, 35 (68.8\%) were asymptomatic.

\section{Factors affecting malaria infection among pregnant women}

From the bi-variable logistic regression, malaria was significantly associated with all of the variables at a significance level of 0.2. However, from the multivariable logistic regression model only age, ITN utilization, consultation about malaria prevention methods during ANC, trimester of pregnancy, and gravidity were significantly associated with malaria infection during pregnancy. For 1 year increase in the age of the pregnant women, the odds of malaria infection was decreased by $22 \%(\mathrm{AOR}=0.78,95 \% \mathrm{CI}: 0.67,0.91)$. The odds of malaria infection was 14.98 times higher among pregnant women who did not utilize ITN compared to their counterparts $(\mathrm{AOR}=14.98,95 \% \mathrm{CI}: 5.24,42.27)$. Pregnant women who had no education about malaria prevention methods during their ANC follow up had 7.15 times increased odds of malaria infection compared to their counterparts (AOR $=7.15,95 \%$ CI: 2.44, 20.96). Women who were in their first trimester of pregnancy had 23.33 times increased odds of having malaria infection compared to mothers on their third trimester $(\mathrm{AOR}=23.33$, 95\% CI: 1.90, 28.20). Women who are in their second trimester of pregnancy also had 7.78 times increased odds of having malaria infection compared to mothers on their third trimester (AOR $=7.78,95 \% \mathrm{CI}: 2.77,21.87$ ). The odds of malaria infection was 5.87 times higher among women who had their second pregnancy compared to multi gravid women $(\mathrm{AOR}=5.87,95 \%$ CI: 1.61, 21.37) (Table 2).

\section{Discussion}

This study assessed the prevalence of malaria infection and associated factors among pregnant women in Sherkole district, Benishangul Gumuz regional state, West Ethiopia. Different studies reported different factors that affect the rate of malaria infection among pregnant women. Our study also assessed socio-demographic, obstetric, and ITN ownership and utilization factors. As a result, Age the woman, ITN utilization, health education about prevention methods during pregnancy, gestational age, and gravidity were found to be significantly associated with malaria infection.

In this study, the prevalence of malaria was found to be $10.2 \%$. This result was higher than studies conducted in Felege Hiwot referral hospital and Addis Zemen health center, Ethiopia (2.83\%) [9], rural district surrounding Arbaminch town, Ethiopia (9.1\%) [11], coastal Ghana (5\%) [19], South-West Nigeria (7.7\%) [5], Southern Laos (8.3\%) [20] and India (5.4\%) [21]. This difference might be attributed to the difference in geographical location among the study areas. For instance, our study was conducted in a malaria-endemic area with a high rate of transmission. Therefore, individuals living in malaria-endemic areas have a greater chance of developing asymptomatic malaria, while those living in low transmission areas have a low chance of being infected, which can lead to a low prevalence of the diseases in such areas. Another reason for the difference could be the inclusion criteria used by the studies because our study included both symptomatic and asymptomatic pregnant women which might increase the prevalence but most of the other studies included only asymptomatic pregnant women. On the other hand, the prevalence in our study was found to be lower than studies conducted in Pawe hospital, Ethiopia (16.3\%) [10], Sudan (13.7\%) [6], Nigeria (41.6\%) [4], Malawi (19.6\%) [8] Burkinafaso (18.1\%) [7] and a systematic review and metaanalysis in Ethiopia 12.7\% [22]. It is also found to be much 
Table 1 Socio demographic characteristics and malaria prevention methods in Sherkole district, West Ethiopia, 2018

\begin{tabular}{|c|c|c|}
\hline Description & Frequency & Percent (\%) \\
\hline \multicolumn{3}{|l|}{ Age group (Years) } \\
\hline $15-19$ & 14 & 2.8 \\
\hline $20-24$ & 87 & 17.5 \\
\hline $25-29$ & 208 & 41.8 \\
\hline $30-34$ & 122 & 24.5 \\
\hline$>=35$ & 67 & 13.5 \\
\hline \multicolumn{3}{|l|}{ Marital status } \\
\hline Married & 492 & 98.8 \\
\hline Others $^{a}$ & 6 & 1.2 \\
\hline \multicolumn{3}{|l|}{ Occupation } \\
\hline Farmer and traditional gold mining & 482 & 96.8 \\
\hline Civil servants & 12 & 2.4 \\
\hline Merchant & 4 & 0.8 \\
\hline \multicolumn{3}{|l|}{ Education } \\
\hline No formal education & 384 & 77.1 \\
\hline Primary & 109 & 21.9 \\
\hline Others $^{b}$ & 5 & 1 \\
\hline \multicolumn{3}{|l|}{ ITN ownership } \\
\hline Yes & 478 & 96 \\
\hline No & 20 & 4 \\
\hline \multicolumn{3}{|l|}{ ITN Utilization } \\
\hline Yes & 354 & 71.3 \\
\hline No & 93 & 18.7 \\
\hline \multicolumn{3}{|l|}{ Use IRS in the last 12 months } \\
\hline Yes & 485 & 97.8 \\
\hline No & 13 & 2.2 \\
\hline \multicolumn{3}{|c|}{ Consult and given health education about malaria prevention and control method } \\
\hline Yes & 431 & 86.5 \\
\hline No & 67 & 13.5 \\
\hline \multicolumn{3}{|l|}{ Gravidity } \\
\hline Primigravidae & 51 & 10.2 \\
\hline Secundigravidae & 124 & 24.9 \\
\hline Multigravidae & 323 & 64.9 \\
\hline \multicolumn{3}{|l|}{ Trimester } \\
\hline 1 st & 15 & 3 \\
\hline 2nd & 191 & 38.4 \\
\hline $3 r d$ & 292 & 58.6 \\
\hline \multicolumn{3}{|l|}{ previous place of delivery } \\
\hline At Health facility & 296 & 66.2 \\
\hline At home & 151 & 33.8 \\
\hline
\end{tabular}


Table 1 Socio demographic characteristics and malaria prevention methods in Sherkole district, West Ethiopia, 2018 (Continued)

\begin{tabular}{llc}
\hline Description & Frequency & Percent (\%) \\
\hline ANC visit & & 67 \\
No ANC follow up & 163 & 13.5 \\
1 time & 158 & 32.8 \\
2 time & 94 & 31.8 \\
3 time & 15 & 18.9 \\
$4+$ time & & 3 \\
\hline
\end{tabular}

ANC Antenatal care, IRS Indoor Residual Spray

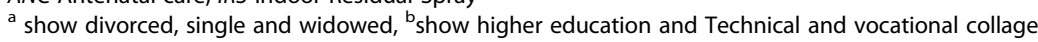

lower than the findings from two studies conducted in Nigeria which showed the prevalence to be $58 \%$ [23] and $59.9 \%$ [24]. This difference may be due to better implementation of improved malaria interventions including increased coverage in the distribution of Long Lasting Insecticide Treated Nets (LLINs), and indoor residual spraying in our study area which showed 96 and $81.3 \%$ of respondents own and utilize ITN, respectively. Almost all (98\%) of participants in our study area also lived in residual sprayed households. Therefore, these interventions might reduce the malaria burden in the study area.
Another possible reason for the low prevalence in our study could be, the study was done during the low malaria transmission season (July - August). However, the major transmission for malaria occurs between September and December.

In this study, $90.2 \%$ of the cases were caused by P.falciparum species. This result was in line with the study conducted in tropical Africa which showed $80-95 \%$ of malaria infections are caused by $P$. falciparum [19]. However, our result was higher than the national prevalence reports of the species which was $60-70 \%$ [25]. This

Table 2 Multivariable analyses of factors associated with malaria infection among pregnant women in Sherkole district, Benishangul Gumuz regional state, West Ethiopia, 2018

\begin{tabular}{|c|c|c|c|c|c|}
\hline \multirow{2}{*}{$\begin{array}{l}\text { Explanatory } \\
\text { variable }\end{array}$} & \multicolumn{2}{|c|}{ Malaria status } & \multirow[t]{2}{*}{ Crud OR(95\%Cl) } & \multirow{2}{*}{$\begin{array}{l}\text { Adjusted OR } \\
(95 \% \mathrm{Cl})\end{array}$} & \multirow{2}{*}{$\begin{array}{l}\mathrm{P} \text { - } \\
\text { value }\end{array}$} \\
\hline & Positive(\%) & $\overline{\text { Negative(\%) }}$ & & & \\
\hline Age & & & $0.74(0.681,0.812)$ & $0.78(0.67,0.91)$ & 0.001 \\
\hline \multicolumn{6}{|l|}{ ITN ownership } \\
\hline Yes & $42(8.8)$ & $436(91.2)$ & 1 & 1 & \\
\hline No & $9(45.0)$ & $11(55.0)$ & $8.49(3.33,21.66)$ & $0.49(0.09,2.60)$ & 0.401 \\
\hline \multicolumn{6}{|l|}{ ITN utilization } \\
\hline Yes & $17(4.2)$ & $388(95.8)$ & 1 & 1 & \\
\hline No & 34 (36.6) & $59(63.4)$ & $13.15(6.91,25.03)$ & $14.89(5.24,42.27)$ & 0.000 \\
\hline \multicolumn{6}{|l|}{ ANC follow up } \\
\hline Yes & $28(6.5)$ & $404(93.5)$ & 1 & 1 & \\
\hline No & $23(34.9)$ & $43(65.1)$ & $7.72(4.09,14.56)$ & $0.91(0.27,3.12)$ & 0.887 \\
\hline \multicolumn{6}{|c|}{ consultation and education about the prevention methods of malaria during ANC } \\
\hline Yes & $24(5.57)$ & $407(94.4)$ & 1 & 1 & \\
\hline No & $27(40.3)$ & $40(59.7)$ & $11.45(6.04,21.68)$ & $7.15(2.44,20.96)$ & 0.000 \\
\hline \multicolumn{6}{|l|}{ Trimester } \\
\hline 1 st & $3(20.0)$ & $12(80.0)$ & $8.88(2.08,37.73)$ & $23.33(1.90,28.20)$ & 0.014 \\
\hline 2nd & $40(20.9)$ & $151(79.1)$ & $9.4(4.29,20.6)$ & $7.78(2.77,21.87)$ & 0.000 \\
\hline $3 r d$ & $8(2.7)$ & $284(97.3)$ & 1 & 1 & \\
\hline \multicolumn{6}{|l|}{ Gravidity } \\
\hline Premigravidea & $12(23.5)$ & $39(76.5)$ & $12.11(4.67,31.47)$ & $0.95(0.14,6.56)$ & 0.963 \\
\hline Secundgravidea & $31(25.0)$ & $93(75.0)$ & $13.13(5.83,29.53)$ & $5.87(1.61,21.37)$ & 0.007 \\
\hline Multigravidea & $8(2.5)$ & $315(97.5)$ & 1 & 1 & \\
\hline
\end{tabular}


high proportion of this malaria species in our study is a clear implication that there is a need for aggressive prevention and control of the diseases, especially among pregnant women. Because $P$. falciparum causes the most severe form of the disease and it can cause devastating complications not only for the mother but also for the fetus. This result also implies that there is a need for early screening of pregnant women for early detection and treatment of the cases to prevent possible complications. On the other hand, the proportion of malaria cases caused by P.falciparum in our study was lower than the WHO malaria 2017 report which revealed over $99 \%$ of malaria cases were due to P.falciparum [1]. The possible reason for these variations might be due to marked seasonal, inter-annual, and spatial variability. It may also be due to large differences in climate (temperature, rainfall, and relative humidity), human settlement, and population movement patterns.

In this study mothers with an increased age were found to have lower odds of developing malaria infection. This is in line with studies conducted in different tropical African countries [5, 17] which reported pregnant women of young age are at the greatest risk of malaria infection, as well as having the highest parasite densities. This may be attributed to mothers with increased age have better exposure to health services and gain a good awareness about the disease and the ways of prevention. Also, due to previous frequent malaria exposures, older aged mothers might develop immunity to malaria. However, according to the studies conducted in rural surroundings of Arbaminch Town, Ethiopia [11], and Sudan [6], age had no significant association with malaria infection.

According to our study, pregnant women who were in the second trimester of pregnancy were at increased odds of developing malaria infection compared to mothers in the third trimester. Besides, and women who were gravidae II have increased odds of malaria infection compared to the multi gravid. Similar results were found from studies done in subSaharan Africa countries [7, 11, 18], which showed a higher risk of malaria infection among primigravidae and gravida two than multigravidae. Low risk of malaria among multigravidae mothers may be associated with the development of pre-immunity to malaria with increased gravidity and previous exposures. It might be also linked to infection-specific immunological factors. Some Plasmodium-infected erythrocytes sequester/arrest in the maternal placenta by producing surface antigens mainly variant surface antigens that adhere to chondroitin sulphate-A (CSA) receptors expressed by syncytiotrophoblasts in the placenta. These antibodies are associated with protection against placental infection. Therefore, primigravidae and secundigravidae mothers lack these antiadhesion antibodies against CSA binding parasites, which develop only after successive pregnancies and this makes them more susceptible to infection [26].
In our study, getting a consultation and health education about malaria preventive methods during ANC follow up significantly decreased the odds of developing malaria infection during pregnancy. A similar association was found in studies conducted different parts of Ethiopia [27, 28]. Health education and consultation specifically on prevention and control program of malaria during pregnancy ensures the use of antimalarials and other intervention measures effectively.

In this study, not using ITN increases the odds of developing malaria infection during pregnancy. Indeed, WHO, $\mathrm{MoH}$, and presidents malaria initiatives (PMI) have advocated for a three-pronged approach to tackle malaria and one of the strategies is the use of ITN $[1,25,29]$. This study's finding was also in agreement with the study conducted in Malawi [8], Nigeria [30], and Arbaminch, Ethiopia [11], which showed that the use of bed nets has a significant impact on decreasing malaria infection. The possible explanation for this association could be ITNs effectively reduce human-mosquito contact which can prevent diseases.

Since our study used a cross-sectional study design, it does not show a direct temporal relationship. Though using PCR and blood film microscopy may have higher sensitivity, we could not do these tests because the study is done in rural areas and there is no electricity in the area. Therefore, the result of this study could be affected by the inherent performance of the RDT utilized.

\section{Conclusion}

The prevalence of malaria infection among pregnant women was relatively low in Sherkole district and P. falciparum is the most predominant Plasmodium species in the area. Age of respondents, ITN use, gravidity, gestational age, and health education about malaria prevention methods during ANC had a significant association with malaria infection. Health professionals should give health education about malaria prevention methods during ANC and they should also give special attention to those pregnant women with the identified risk factors. Besides, further research is recommended by using more sensitive diagnostic methods like PCR and blood film microscopy for the diagnosis of malaria.

\section{Abbreviations \\ AOR: Adjusted Odds Ratio; ANC: Antenatal Care; BGRS: Benishangul Gumuz Regional state; (CSA): Chondroitin Sulphate-A; Cl: Confidence Interval; ITN: Insecticide Treated Net; LLINs: Long Lasting Insecticide Treated Nets; $\mathrm{MoH}$ : Ministry of Health; OR: Odds Ratio; PMI: Presidents Malaria Initiatives; RDT: Rapid Diagnostic Test; WHO: World Health Organization}

\section{Acknowledgments}

We would like to express our deepest thanks to the University of Gondar College of Medicine and Health Sciences and Health Officer Department, for facilitating the research work. We also want to thank all pregnant women who participated in this study for their contribution. 


\section{Authors' contributions}

All authors actively participate in the conception and design, acquisition of data, or analysis and interpretation of data. GBG, HFW, and AGB critically revise the manuscript. All authors read and approved the final version of the manuscript.

\section{Funding}

Not applicable.

\section{Availability of data and materials}

The data upon which the result based could be accessed a reasonable request.

\section{Ethics approval and consent to participate}

Ethical clearance was obtained from the Ethical review board of the University of Gondar and the permission letter was also obtained from Benishangul Gumuz regional state administration Health Bureau. Then this letter was delivered to Sherkole district Health office and the respected villages. The purpose and importance of the study were explained to the participants and since the majority of our study participants cannot read and write, verbal consent was obtained from each participant above the age of 18. Assent was also obtained for participants below the age of 18 from their parents or guardian. Pregnant women who tested positive were linked to the nearby health center for treatment. Confidentiality of the information was maintained by omitting their names and personal identification.

\section{Consent for publication}

Not applicable.

\section{Competing interests}

All authors declared that they have no competing interests.

\section{Author details}

'Benishangul Gumuz Regional State Health Bureau, Asosa, Ethiopia. 2 Department of Epidemiology and Biostatistics, Institute of Public Health, College of Medicine and Health Sciences, University of Gondar, Gondar, Ethiopia.

Received: 29 May 2019 Accepted: 26 July 2020

Published online: 05 August 2020

\section{References}

1. WHO. World malaria report 2017 World health organization 2017.

2. WHO. World malaria report 2016. 2016.

3. Steketee RWNB, Parise MEMC. The burden of malaria in pregnancy in malaria-endemic areas. Am J Trop Med Hyg. 2001;64:28-35.

4. Kagu M, Kawuwa M, Gadzama G. Anaemia in pregnancy: a cross-sectional study of pregnant women in a Sahelian tertiary hospital in northeastern Nigeria. J Obstet Gynaecol. 2007;27(7):676-9.

5. Agomo CO, Oyibo WA, Anorlu Rl, Agomo PU. Prevalence of malaria in pregnant women in Lagos, south-West Nigeria. Korean J Parasitol. 2009; 47(2):179.

6. Adam I, Khamis AH, Elbashir MI. Prevalence and risk factors for Plasmodium falciparum malaria in pregnant women of eastern Sudan. Malar J. 2005:4(1):18.

7. Cisse M, Sangare I, Lougue G, Bamba S, Bayane D, Guiguemde RT. Prevalence and risk factors for Plasmodium falciparum malaria in pregnant women attending antenatal clinic in Bobo-Dioulasso (Burkina Faso). BMC Infect Dis. 2014;14(1):631

8. Boudová S, Divala T, Mawindo P, Cohee L, Kalilani-Phiri L, Thesing P, et al. The prevalence of malaria at first antenatal visit in Blantyre, Malawi declined following a universal bed net campaign. Malar J. 2015;14(1):422.

9. Asmamaw T, Alemu A, Alemu A, Unakal C. Prevalence of malaria and HIV among pregnant women attending antenatal clinics at felege hiwot referral hospital and Addis zemen health center. Int J Life Sci Biotechnol Pharma Res. 2013:2(1-13).

10. Geleta G, Ketema T. Prevalence of malaria and frequency of severe symptoms among pregnant women in Pawe hospital, North Western Ethiopia. Ann Clin Pathol. 2017;5:1109.

11. Nega D, Dana D, Tefera T, Eshetu T. Prevalence and predictors of asymptomatic malaria parasitemia among pregnant women in the rural surroundings of Arbaminch town, South Ethiopia. PLoS One. 2015;10(4): e0123630.

12. Gajida A, lliyasu Z, Zoakah A. Malaria among antenatal clients attending primary health care facilities in Kano state, Nigeria. Annals of African medicine. 2010;9(3)..

13. BJ B. An analysis of malaria in pregnancy in Africa. Bull World Health Organ 1983; 61:1005-1016.

14. WHOAFRG. African Region 2010 malaria report. 2010.

15. WHO. A Strategic Framework for Malaria Prevention and Control During Pregnancy in the African Region, In World Health Organization RegionalOffice for Africa. AFR/MAL/04/01. 2004.

16. Fana SA, Bunza MD, Anka SA, Imam AU, Nataala SU. Prevalence and risk factors associated with malaria infection among pregnant women in a semi-urban community of North-Western Nigeria. Infect Dis Poverty. 2015:4:24.

17. Jäckle MJ, Blumentrath CG, Zoleko RM, Akerey-Diop D, Mackanga J-R, Adegnika $A A$, et al. Malaria in pregnancy in rural Gabon: a cross-sectional survey on the impact of seasonality in high-risk groups. Malar J. 2013;12(1):412.

18. Mario J Jäckle CGB, Rella M Zoleko, Daisy Akerey-Diop, Jean-Rodolphe Mackanga, Ayôla A Adegnika BL, Pierre-Blaise Matsiegui, Peter G Kremsner, Ramharter GMNaM Malaria in pregnancy in rural Gabon: a cross-sectional survey on the impact of seasonality in high-risk groups malaria journal 2013;12:412.

19. Steffen R. debernardis C, Baños A. Travel epidemiology-a global perspective. Int J Antimicrob Agents. 2003;21(2):89-95.

20. Briand V, Le Hesran JY, Mayxay M, Newton PN, Bertin G, Houze S, et al. Prevalence of malaria in pregnancy in southern Laos: a cross-sectional survey. Malar J. 2016:15(1):436.

21. Sohail M, Shakeel S, Kumari S, Bharti A, Zahid F, Anwar S, et al. Prevalence of malaria infection and risk factors associated with Anaemia among pregnant women in Semiurban Community of Hazaribag, Jharkhand, India. Biomed Res Int. 2015:2015:740512.

22. Tegegne $Y$, Asmelash D, Ambachew S, Eshetie S, Addisu A, Jejaw ZA. The prevalence of malaria among pregnant women in Ethiopia: a systematic review and meta-analysis. J Parasitol Res. 2019;8396091.

23. Ifeanyi U, Onyebuchi V, Ukamaka T, Ugochukwu B. Asymptomatic Plasmodium parasitaemia in pregnant Nigerian women : almost a decade after roll Back malaria. Malar J. 2009;24:16-20.

24. Ogbodo S, Nwagha, U., Okaka, A., Ogenyi, S., Okoko, R., \& Nwagha, T. Malaria parasitaemia among pregnant women in a rural community of eastern Nigeria; need for combined measures. Nigerian Journal of Physiological Science. (2009);24(2)..

25. MOH. Four years NMSP of Ethiopia from2014/2015-2019/2020. 2014.

26. FriedM N, Brockman A, Brabin BJ, Duffy PE. Maternalantibodiesblockmalaria. Nature. 1998;95:852

27. Deribew A, Alemseged F, Birhanu Z, Sena L, Tegegn A, Zeynudin A, et al. Effect of training on the use of long-lasting insecticide-treated bed nets on the burden of malaria among vulnerable groups, south-West Ethiopia: baseline results of a cluster randomized trial. Malar J. 2010;9(1):1303-13.

28. Belay M DW. Use of insecticide treated nets by pregnant women and associated factors in a pre-dominantly rural population in northern Ethiopia. Trop Med Int Health. 2008;13(10).

29. INITIATIVE PSM, ETHIOPIA. Malaria Operational Plan FY 20172017.

30. Oyibo COAaWA. Factors associated with risk of malaria infection among pregnant women in Lagos. Nigeria: Infect Dis Poverty; 2013.

\section{Publisher's Note}

Springer Nature remains neutral with regard to jurisdictional claims in published maps and institutional affiliations.

Ready to submit your research? Choose BMC and benefit from:

- fast, convenient online submission

- thorough peer review by experienced researchers in your field

- rapid publication on acceptance

- support for research data, including large and complex data types

- gold Open Access which fosters wider collaboration and increased citations

- maximum visibility for your research: over $100 \mathrm{M}$ website views per year

At BMC, research is always in progress.

Learn more biomedcentral.com/submissions 